\title{
ASCA and RXTE Upper Limits to Pulsed Emissions from PSR B1951+32
}

\author{
Hsiang-Kuang Chang and Tz-Feng Guo \\ Department of Physics, National Tsing Hua University, Hsinchu 300, \\ Taiwan, $R O C$
}

\begin{abstract}
We report upper limits to pulsed X-ray emissions from PSR B1951+32 in $0.5-250 \mathrm{keV}$ based on observations of ASCA in 1993 and RXTE in 1996. Together with earlier ROSAT report, these upper limits strongly suggest a thermal pulsed component below about $1 \mathrm{keV}$. These upper limits also indicate a spectral bending occurring in $100 \mathrm{keV}-3$ $\mathrm{MeV}$, which favors an outer-magnetosphere origin for gamma-ray emissions from PSR B1951+32.
\end{abstract}

\section{In Search of Pulsations}

Although located in a well studied supernova remnant CTB 80, X-ray emission from PSR B1951+32 still remains uncertain. ROSAT observation of PSR B1951+32 reported an estimated pulsed fraction of 0.35 and an overall (pulsed plus unpulsed) spectrum following a power law with a photon spectral index of $-1.6 \pm 0.2$ (Safi-Harb, Ögelman, \& Finley 1995). To date, there is no published spectrum for the pulsed component.

In this paper we report results of searching for pulsation in ASCA/GIS and RXTE/PCA, /HEXTE data. After screening, data used in analysis are all about $23 \mathrm{ksec}$ for each of these three data sets. Although no pulsation is firmly found, estimated upper limits, as plotted in Figure 1, are low enough to reveal important information as discussed below.

\section{Discussion}

It is clear from Figure 1 that the ASCA/GIS upper limits, concordantly with RXTE upper limits, necessitate a steep drop-off at around $1 \mathrm{keV}$ for the pulsed component. This pulsed emission must be much softer than the reported power law in ROSAT data, probably with a thermal characteristic. Furthermore, it also indicates a nonthermal, unpulsed emission around $2 \mathrm{keV}$ and extending up to an undetermined energy in hard X-rays. This is a separate component from those pulsed gamma-ray emissions of this pulsar. Such an unpulsed nonthermal emission is not expected in the model proposed by Cheng \& Zhang (1999).

On the other hand, these upper limits show that the overall spectrum from gamma-ray downwards must start flattening from between $100 \mathrm{keV}$ and $3 \mathrm{MeV}$ with a photon spectral index significantly deviating from -1.5 . Such a spectral bending can be used to infer the location and mechanism of gamma-ray 


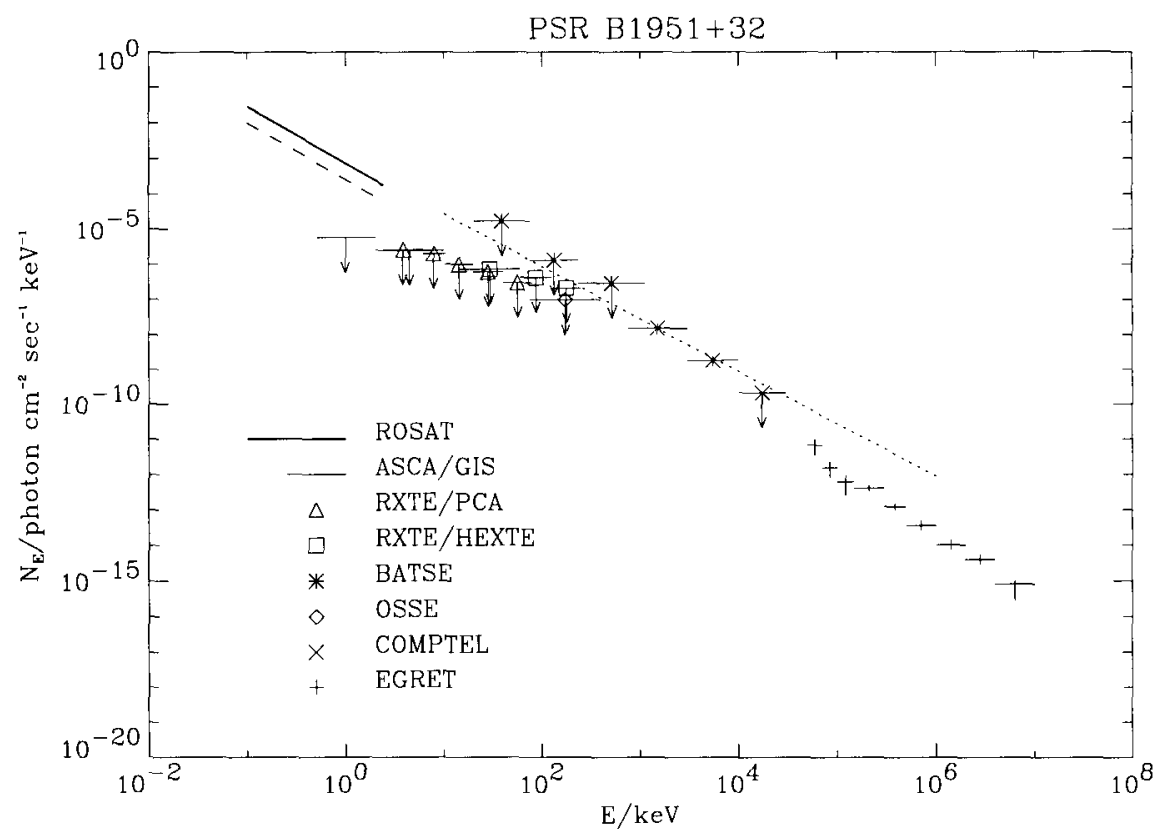

Figure 1. Spectrum of PSR B1951+32 from $0.1 \mathrm{keV}$ up to $10 \mathrm{GeV}$. The ROSAT data is for the X-ray point source including pulsed and unpulsed components. The dashed line is the solid line times 0.35 , the reported pulsed fraction of this point source. The dotted line is a power law with a photon spectral index $-3 / 2$ passing through the COMPTEL data point in $0.75-3.0 \mathrm{MeV}$. Upper limits are at a $2-\sigma$ level for ASCA/GIS, RXTE/PCA, RXTE/HEXTE, OSSE, and COMPTEL, and $3-\sigma$ for BASTE.

emissions, provided a steady cooling population of charges is the source of these gamma-rays, which is very likely the case as indicated by power law spectra of all EGRET pulsars (Chang \& Ho 1997, 1999). With such a bending in $100 \mathrm{keV}$ - $3 \mathrm{MeV}$ for PSR B1951+32, it is found that the emission site is close to the light cylinder and synchrotron radiation is the dominant mechanism.

\section{References}

Chang, H.-K., \& Ho, C. 1997, ApJ, 479, L125

Chang, H.-K., \& Ho, C. 1999, ApJ, 510, 414

Cheng, K. S., \& Zhang, L. 1999, ApJ, 515, 337

Safi-Harb, S., Ögelman, H., \& Finley, J.P. 1995, ApJ, 439, 722 